\title{
FINITE 2-COMPLEXES WITH INFINITELY-GENERATED GROUPS OF SELF-HOMOTOPY-EQUIVALENCES
}

\author{
A. M. BRUNNER AND J. G. RATCLIFFE
}

\begin{abstract}
Examples of finite 2-dimensional aspherical cell complexes are given whose group of homotopy classes of self-homotopy-equivalences is infinitely generated.
\end{abstract}

In a recent paper [11], Darryl McCullough gave an example of a finite, 4-dimensional, aspherical cell complex whose group of homotopy classes of self-homotopyequivalences is infinitely generated, and asked if there is a 2-dimensional example. In this paper, we give an infinite family of finite, 2-dimensional, aspherical cell complexes each of whose group of homotopy classes of self-homotopy-equivalences is infinitely generated.

McCullough's example is based on Lewin's [8] example of a finitely presented group with an infinitely generated automorphism group. Our examples are based on recent examples, given by the first named author [3], of one-relator groups with infinitely-generated automorphism group.

Let $X$ be a path connected space with base point. We let $\mathcal{E}(X)$ denote the group of based homotopy classes of self-homotopy-equivalences, and $\mathscr{F}(X)$ the group of free homotopy classes of self-homotopy-equivalences. If $X$ is an aspherical CWcomplex with fundamental group $G$, then $\mathcal{E}(X) \cong$ Aut $G$ and $\mathscr{F}(X) \cong$ Out $G$ [20, pp.225-226]. If $G$ is finitely generated, then Aut $G$ is finitely generated if and only if Out $G$ is finitely generated. Thus, if $X$ is a finite, aspherical complex, then $\mathscr{E}(X)$ is finitely generated if and only if $\mathscr{F}(X)$ is finitely generated.

EXAMPLE 1. Let $n$ be a natural number greater than one, and consider the group

$$
G(n)=\left(a, t ; t^{-1} a^{-1} t a^{n} t^{-1} a t=a\right) .
$$

The groups $\{G(n) \mid n=2,3, \ldots\}$ are mutually nonisomorphic, since $G(n)$ abelianizes to $\mathbf{Z}_{n-1} \oplus \mathbf{Z}$. In [3], it is shown that Out $G(n) \cong \mathbf{Z}\left[\frac{1}{n}\right]$ - the additive group of rational numbers with denominator a nonnegative power of $n$. The group $\mathbf{Z}\left[\frac{1}{n}\right]$ is infinitely generated, since it is locally cyclic, but not cyclic. Thus Out $G(n)$ and Aut $G(n)$ are infinitely generated.

Let $X(n)$ be the 2-dimensional cell-complex which models the presentation of $G(n)$. Then $X(n)$ is a finite cell-complex (one 0-cell, two 1-cells and one 2-cell) with

Received by the editors March 6, 1981 and, in revised form, February 8, 1982.

1980 Mathematics Subject Classification. Primary 55P10; Secondary 57M20.

Key words and phrases. self-homotopy-equivalence, 2-dimensional complex, infinitely-generated group, aspherical complex, automorphism group, lst $k$-invariant. 
fundamental group isomorphic to $G(n)$. As $X(n)$ has only one 2-cell, it follows from a lemma of Cockcroft [4] (see also [5]) that $X(n)$ is aspherical. Therefore, $\mathscr{F}(X(n)) \cong$ $\operatorname{Out}(G(n)) \cong \mathbf{Z}\left[\frac{1}{n}\right]$. Thus $\mathscr{F}(X(n))$ and $\mathcal{E}(X(n))$ are not finitely generated.

At this point we would like to thank the referee for asking the following interesting question.

Question 1. Is there a finite two-dimensional complex $X$ with $\operatorname{Aut}\left(\pi_{1}(X)\right)$ finitelygenerated but $\mathcal{E}(X)$ not finitely-generated?

To see what answering this question would entail, let $\mathcal{E}^{1}(X)$ and $G(X)$ be the kernel and image respectively of the evaluation homomorphism

$$
\mathcal{E}(X) \rightarrow \operatorname{Aut}\left(\pi_{1}(X)\right) .
$$

Then we have an exact sequence

$$
1 \rightarrow \mathcal{E}^{1}(X) \rightarrow \mathcal{E}(X) \rightarrow G(X) \rightarrow 1 .
$$

If $\mathcal{E}(X)$ is not finitely generated then either $G(X)$ or $\mathcal{E}^{1}(X)$ is not finitely generated. This suggests Questions 2 and 3.

Question 2. Is there a finite two-dimensional complex $X$ with $\operatorname{Aut}\left(\pi_{1}(X)\right)$ finitelygenerated but $G(X)$ not finitely-generated?

This question appears to be difficult, since not much is known about the relation of $G(X)$ to Aut $\left(\pi_{1}(X)\right)$. What is known is that if $X$ is aspherical or if $X$ has only one 2-cell (Jajodia [7]) then $G(X)=\operatorname{Aut}\left(\pi_{1}(X)\right)$, while Schellenberg [15] and Sieradski [18] have shown that $G(X)$ may be a proper subgroup of $\operatorname{Aut}\left(\pi_{1}(X)\right)$ when $\pi_{1}(X)$ is a noncyclic finite abelian group.

Question 3. Is there a finite 2-dimensional complex $X$ such that $\mathcal{E}^{1}(X)$ is not finitely generated?

Before we answer this question below, we need to analyze $\mathcal{E}^{l}(X)$ when $X$ is a finite 2-dimensional complex. Consider the evaluation homomorphism $\mathcal{E}^{1}(X) \rightarrow$ Aut $_{\pi_{1}}\left(\pi_{2}(X)\right)$. It follows from equation (1) in Adams [1] that the image is the subgroup Aut $\pi_{1}^{k}\left(\pi_{2}(X)\right)$ of all $\pi_{1}$-automorphisms of $\pi_{2}(X)$ which fix $k(X)$, the first $k$-invariant of $X$ in $H^{3}\left(\pi_{1}(X), \pi_{2}(X)\right)$; moreover, Schellenberg [14] has shown that the kernel is isomorphic to $H^{2}\left(\pi_{1}(X), \pi_{2}(X)\right)$. Thus we have an exact sequence

$$
0 \rightarrow H^{2}\left(\pi_{1}(X), \pi_{2}(X)\right) \rightarrow \mathbb{E}^{1}(X) \rightarrow \operatorname{Aut}_{\pi_{1}}^{k}\left(\pi_{2}(X)\right) \rightarrow 1 .
$$

In order to compute $\AA^{-1}(X)$, it would be convenient to have $k(X)=0$, since then $\operatorname{Aut}_{\pi_{1}}^{k}\left(\pi_{2}(X)\right)=\operatorname{Aut}_{\pi_{1}}\left(\pi_{2}(X)\right)$. This certainly is the case if the cohomological dimension of $\pi_{1}(X)$ is at most two, since $k(X)$ lives in a 3-dimensional cohomology group of $\pi_{1}(X)$. The following theorem says that, in fact, the converse is true.

THEOREM 1. Let $k(X)$ be the first $k$-invariant of a 2-dimensional $C W$-complex $X$. Then $k(X)=0$ if and only if the cohomological dimension of $\pi_{1}(X)$ is at most two.

Proof. Suppose $k(X)=0$. Recall that $k(X)$ is represented by the crossed sequence [10]

$$
0 \rightarrow \pi_{2}(X) \rightarrow \pi_{2}\left(X, X^{1}\right) \stackrel{\partial_{2}}{\rightarrow} \pi_{1}\left(X^{1}\right) \rightarrow \pi_{1}(X) \rightarrow 1
$$


where $X^{1}$ is the 1-skeleton of $X$. Let $N=\operatorname{Im} \partial_{2}$. Then $N$ is the group of relations of the presentation for $\pi_{1}(X)$ prescribed by the cell structure of $X$. By Theorem 8.1 of [12], the crossed extension

$$
0 \rightarrow \pi_{2}(X) \rightarrow \pi_{2}\left(X, X^{1}\right) \rightarrow N \rightarrow 1
$$

operator splits. As $\pi_{2}\left(X, X^{1}\right)$ is a free crossed module, we have that $N$ is a projective crossed module [13]. This implies that the relation module $N_{\mathrm{ab}}$ is a projective $\pi_{1}(X)$-module. Therefore, cd $\pi_{1}(X) \leqslant 2$ by Serre [17, p. 90].

Next, we give an example which may possibly give an affirmative answer to Question 1.

EXAmple 2. Let $X=S^{1} \vee S^{2} \vee S^{2}$. Then $\pi_{1}(X) \cong \mathbf{Z}$ and $\pi_{2}(X) \cong \mathbf{Z}(\mathbf{Z}) \oplus \mathbf{Z}(\mathbf{Z})$. Clearly, $G(X)=\operatorname{Aut}(\mathbf{Z})= \pm 1$. As $\operatorname{cd} \mathbf{Z}=1$, we have that $H^{i}\left(\pi_{1}(X), \pi_{2}(X)\right)=0$ for $i=2,3$; therefore, $\mathcal{E}^{\prime}(X) \cong \operatorname{Aut}_{\pi_{1}}\left(\pi_{2}(X)\right) \cong \mathrm{GL}_{2}(\mathbf{Z}(\mathbf{Z}))$. Thus we have an exact sequence

$$
1 \rightarrow \mathrm{GL}_{2}(\mathbf{Z}(\mathbf{Z})) \rightarrow \mathcal{E}(X) \rightarrow \pm 1 \rightarrow 1 .
$$

In particular, $\mathcal{E}(X)$ is finitely generated if and only if $\mathrm{GL}_{2}(\mathbf{Z}(\mathbf{Z})$ ) is finitely generated. Note that $\mathbf{Z}(\mathbf{Z})$ is just the ring $\mathbf{Z}\left[t, t^{-1}\right]$ of Laurent polynomials in one variable $t$. Surprisingly, it is unknown whether or not $\operatorname{GL}_{2}\left(\mathbf{Z}\left[t, t^{-1}\right]\right)$ is finitely generated. Thus we ask

Question 4. Is the group $\mathrm{GL}_{2}\left(\mathbf{Z}\left[t, t^{-1}\right]\right)$ infinitely generated?

Note that an affirmative answer to Question 4 and Example 2 gives an affirmative answer to Question 1. Also, see Lemma 1 below.

Let $\mathcal{E}^{2}(X)$ be the kernel of the evaluation homomorphism

$$
\mathcal{E}^{1}(X) \rightarrow \operatorname{Aut}_{\pi_{1}}\left(\pi_{2}(X)\right) \text {. }
$$

Then $\mathcal{E}^{2}(X)$ is the group of homotopy classes of self-homotopy-equivalences of $X$ which induce the identity on both $\pi_{1}(X)$ and $\pi_{2}(X)$. Schellenberg remarked in [14] that he knew of no example of a finite 2-complex $X$ such that $\mathcal{E}^{2}(X)$ is nontrivial. Next, we give two such examples, one for which $\mathcal{E}^{2}(X)$ is finitely generated and the other for which $\mathcal{E}^{2}(X)$ is infinitely generated.

EXAmple 3. Let $X=T^{2} \vee S^{2}$ where $T^{2}$ is the torus. Then $\pi_{1}(X) \cong \mathbf{Z} \oplus \mathbf{Z}$ and $\pi_{2}(X) \cong \mathbf{Z}\left(\pi_{1}(X)\right)$. By Poincaré duality

$$
H^{2}\left(\pi_{1}, \pi_{2}\right) \cong H_{0}\left(\pi_{1}, \pi_{2}\right) \cong \mathbf{Z}
$$

Therefore, $\mathcal{E}^{2}(X)$ is infinite cyclic. Marshall Cohen described to us a map representing a generator of $\mathcal{E}^{2}(X)$ as gotten by pinching a 2-sphere off $T^{2}$ at the base point and mapping it by a degree one map to the wedged $S^{2}$. It is interesting to note that this map induces a nontrivial automorphism of $\mathrm{H}_{2}(X)$.

To complete the analysis of $\mathcal{E}(X)$, notice that the first $k$-invariant of $X$ is zero since $\mathbf{Z} \oplus \mathbf{Z}$ is a 2-dimensional group. Therefore, $\operatorname{Aut}_{\pi_{1}}^{k}\left(\pi_{2}(X)\right)$ is isomorphic to the group of units of $\mathbf{Z}\left(\pi_{1}(X)\right)$. By Theorem 13 of Higman [6] the ring $\mathbf{Z}\left(\pi_{1}(X)\right)$ has only trivial units. Thus $\mathscr{E}(X)$ is determined up to extension by the exact sequences

$$
\begin{aligned}
& 1 \rightarrow \mathcal{E}^{1}(X) \rightarrow \mathcal{E}(X) \rightarrow \mathrm{GL}_{2}(\mathbf{Z}) \rightarrow 1, \\
& 0 \rightarrow \mathbf{Z} \rightarrow \mathcal{E}^{1}(X) \rightarrow \pm \mathbf{Z} \oplus \mathbf{Z} \rightarrow 0 .
\end{aligned}
$$

In particular, $\mathcal{E}^{2}(X), \mathcal{E}^{1}(X)$ and $\mathcal{E}(X)$ are all finitely generated. 
EXAmple 4. Let $X=K \vee S^{2}$ where $K$ is the finite 2-complex which models the presentation $\left(x, y ; x^{2}=y^{3}\right)$ of the trefoil knot group $G$. Then $\pi_{1}(X) \cong G, \pi_{2}(X) \cong$ $\mathbf{Z}(G)$ and $\mathcal{E}^{2}(X) \cong H^{2}(G ; \mathbf{Z}(G))$.

The center of $G$ is generated by $x^{2}$; therefore, we have a central extension

$$
0 \rightarrow \mathbf{Z} \rightarrow G \rightarrow Q \rightarrow 1
$$

where $Q=\mathbf{Z}_{2} * \mathbf{Z}_{3}$.

Next, feed this extension into the Lyndon-Hochschild-Serre spectral sequence to compute $H^{2}(G, \mathbf{Z}(G))$. One computes easily that $H^{2}(G, \mathbf{Z}(G)) \cong E_{2}^{1,1}$. By Poincaré duality we have

$$
H^{1}(\mathbf{Z}, \mathbf{Z}(G)) \cong H_{0}(\mathbf{Z}, \mathbf{Z}(G)) \cong \mathbf{Z}(Q) .
$$

Therefore, $H^{2}(G, \mathbf{Z}(G)) \cong H^{1}(Q, \mathbf{Z}(Q))$. It is well known [19] that $H^{1}(Q, \mathbf{Z}(Q))$ is a free abelian group of countably infinite rank. Therefore, $\mathcal{E}^{2}(X)$ is a free abelian group of countably infinite rank.

The commutator subgroup of $G$ is free of rank 2 and $G_{\mathrm{ab}} \cong \mathbf{Z}$; therefore, $G$ is indicable throughout by the lemma in the appendix of [6]. Hence, $\mathbf{Z}(G)$ has only trivial units. Thus, we have an exact sequence

$$
0 \rightarrow \mathcal{E}^{2}(X) \rightarrow \mathcal{E}^{1}(X) \rightarrow \pm G \rightarrow 1 .
$$

At this point the reader should be wondering whether or not $\mathcal{E}^{1}(X)$ is infinitely generated. From the cochain complex obtained from the Lyndon resolution [9] of the one-relator group $G$, one sees that $H^{2}(G, \mathbf{Z}(G))$ is a cyclic right $G$-module; moreover, one sees from the description of $\mathcal{E}^{1}(X)$ in [14] that the action of $\pm G$ on $\mathcal{E}^{2}(X)$ induced by conjugation in $\mathcal{E}^{1}(X)$ corresponds to right translation in $H^{2}(G, \mathbf{Z}(G))$. This implies easily that $\mathcal{E}^{1}(X)$ is finitely generated.

Finally, we have an exact sequence

$$
1 \rightarrow \mathcal{E}^{1}(X) \rightarrow \mathcal{E}(X) \rightarrow \text { Aut } G \rightarrow 1 .
$$

The group Aut $G$ is finitely generated [16]; therefore, $\mathcal{E}(X)$ is finitely generated.

Before we give our last example, which answers Question 3, we need to prove the following lemma.

LEMMA 1. The group $\mathrm{GL}_{2}\left(\mathbf{Z}\left[t_{1}, t_{1}^{-1}, \ldots, t_{n}, t_{n}^{-1}\right]\right)$ is infinitely generated if and only if

$$
\mathrm{SL}_{2}\left(\mathbf{Z}\left[t_{1}, t_{1}^{-1}, \ldots, t_{n}, t_{n}^{-1}\right]\right)
$$

is infinitely generated.

Proof. Let $G=\mathrm{GL}_{2}\left(\mathbf{Z}\left[t_{1}, t_{1}^{-1}, \ldots, t_{n}, t_{n}^{-1}\right]\right), G_{1}=\mathrm{SL}_{2}\left(\mathbf{Z}\left[t_{1}, t_{1}^{-1}, \ldots, t_{n}, t_{n}^{-1}\right]\right)$, and $U=\mathbf{Z}\left[t_{1}, t_{1}^{-1}, \ldots, t_{n}, t_{n}^{-1}\right]^{*}$. Then $U \cong \mathbf{Z}_{2} \oplus \mathbf{Z}^{n}$ is generated multiplicatively by $-1, t_{1}, \ldots, t_{n}$. Because of the exact sequence

$$
1 \rightarrow G_{1} \rightarrow G \stackrel{\text { Det }}{\rightarrow} U \rightarrow 1
$$

the group $G$ is infinitely generated only if $G_{1}$ is infinitely generated.

Conversely, suppose $G_{1}$ is infinitely generated. Let $G_{2}$ be the subgroup of $G$ consisting of the matrices whose determinant is in the group generated by $t_{1}^{2}, \ldots, t_{n}^{2}$. 
Then there is a retraction $\rho: G_{2} \rightarrow G_{1}$ defined by

$$
\rho(A)=\left(\begin{array}{cc}
u^{-1} & 0 \\
0 & u^{-1}
\end{array}\right) A \quad \text { where } \operatorname{det} A=u^{2} .
$$

Therefore, $G_{2}$ is infinitely generated. But $G_{2}$ is a subgroup of index $2^{n+1}$ in $G$; therefore, $G$ is infinitely generated.

EXAMPLE 5. Let $X=T^{2} \vee S^{2} \vee S^{2}$. Then $\pi_{1}(X) \cong \mathbf{Z} \oplus \mathbf{Z}$ and $\pi_{2}(X) \cong \mathbf{Z}(\mathbf{Z} \oplus \mathbf{Z})$ $\oplus \mathbf{Z}(\mathbf{Z} \oplus \mathbf{Z})$. Therefore, we have an exact sequence

$$
1 \rightarrow \mathcal{E}^{1}(X) \rightarrow \mathcal{E}(X) \rightarrow \mathrm{GL}_{2}(\mathbf{Z}) \rightarrow 1 .
$$

By Poincaré duality

$$
H^{2}\left(\pi_{1}, \pi_{2}\right) \cong H_{0}\left(\pi_{1}, \pi_{2}\right) \cong \mathbf{Z} \oplus \mathbf{Z} .
$$

Therefore, we have an exact sequence

$$
0 \rightarrow \mathbf{Z} \oplus \mathbf{Z} \rightarrow \mathcal{E}^{1}(X) \rightarrow \mathrm{GL}_{2}(\mathbf{Z}(\mathbf{Z} \oplus \mathbf{Z})) \rightarrow 1 .
$$

The ring $\mathbf{Z}(\mathbf{Z} \oplus \mathbf{Z})$ is just $\mathbf{Z}\left[t_{1}, t_{1}^{-1}, t_{2}, t_{2}^{-1}\right]$.

Recently, Bachmuth and Mochizuki [2] have shown that $\mathrm{SL}_{2}\left(\mathbf{Z}\left[t_{1}, t_{1}^{-1}, t_{2}, t_{2}^{-1}\right]\right)$ is infinitely generated. Therefore, by the lemma, $\mathrm{GL}_{2}\left(\mathbf{Z}\left[t_{1}, t_{1}^{-1}, t_{2}, t_{2}^{-1}\right]\right)$ is infinitely generated. This implies that $\mathcal{E}^{l}(X)$ is infinitely generated. However, it is unclear whether or not $\mathcal{E}(X)$ is finitely generated, since $\mathrm{GL}_{2}(\mathbf{Z})$ is finitely generated.

Question 5. Is $\mathcal{E}\left(T^{2} \vee S^{2} \vee S^{2}\right)$ infinitely generated?

\section{REFERENCES}

1. J. F. Adams, Four applications of the self-obstruction invariants, J. London Math. Soc. 31 (1956), $148-159$.

2. S. Bachmuth and $\mathrm{H}$. Y. Mochizuki, $E_{2} \neq \mathrm{SL}_{2}$ for most Laurent polynomial rings, Preprint, Univ. of California at Santa Barbara, 1981.

3. A. M. Brunner, On a class of one-relator groups, Canad. J. Math. 32 (1980), 414-420.

4. W. H. Cockcroft, On two-dimensional aspherical complexes, Proc. London Math. Soc. 4 (1954), $375-384$.

5. E. Dyer and A. T. Vasquez, Some small aspherical spaces, J. Austral. Math. Soc. 16 (1973), 332-352.

6. G. Higman, The units of group-rings, Proc. London Math. Soc. 46 (1940), 231-248.

7. S. Jajodia, On 2-dimensional CW-complexes with a single 2-cell, Pacific J. Math. 80 (1979), 191-203.

8. J. Lewin, A finitely presented group whose group of automorphisms is infinitely generated, J. London Math. Soc. 42 (1967), 610-613.

9. R. C. Lyndon, Cohomology theory of groups with a single defining relation, Ann. of Math. 52 (1950), 650-665.

10. S. Mac Lane and J. H. C. Whitehead, On the 3-type of a complex, Proc. Nat. Acad. Sci. U.S.A. 36 (1950), 41-48.

11. D. McCullough, Finite aspherical complexes with infinitely-generated groups of self-homotopy-equivalences, Proc. Amer. Math. Soc. 80 (1980), 337-340.

12. J. G. Ratcliffe, Crossed extensions, Trans. Amer. Math. Soc. 257 (1980), 73-89.

13. __ Free and projective crossed modules, J. London Math. Soc. 22 (1980), 66-74.

14. B. Schellenberg, The group of homotopy self-equivalences of some compact $C W$-complexes, Math. Ann. 200 (1973), 253-266.

15. , On the self-equivalences of a space with non-cyclic fundamental group, Math. Ann. 205 (1973), 333-344.

16. O. Schreier, Über die Gruppen $A^{a} B^{b}=1$, Abh. Math. Sem. Univ. Hamburg 3 (1924), 167-169.

17. J.-P. Serre, Cohomologie des groupes discrets, Prospects in Mathematics, Ann. of Math. Studies, no. 70, Princeton Univ. Press, Princeton, N. J., pp. 77-132. 
18. A. J. Sieradski, Combinatorial isomorphisms and combinatorial homotopy equivalences, J. Pure Appl. Algebra 7 (1976), 59-95.

19. E. Specker, Die erste Cohomologiegruppe von Überlagerungen und Homotopie-Eigenschaften dreidimensionaler Mannigfaltigkeiten, Comment. Math. Helv. 23 (1949), 303-333.

20. G. W. Whitehead, Elements of homotopy theory, Graduate Texts in Math., vol. 61, Springer-Verlag, New York, Heidelberg and Berlin, 1978.

Department of Mathematics, University of Wisconsin-Parkside, Kenosha, Wisconsin 53140

Department of Mathematics, University of Wisconsin, Madison, Wisconsin 53706

School of Mathematics, The Institute for Advanced Study, Princeton, New Jersey 08540 